Thorax (1954), 9, 58.

\title{
LINGULAR BIOPSY IN MITRAL STENOSIS
}

BY

\author{
J. B. ENTICKNAP, B. B. MILSTEIN, AND CHARLES BAKER \\ From Guy's Hospital, London
}

(RECEIVED FOR PUBLICATION AUGUST 17, 1953)

Interest in the pathology of the lung vessels in mitral stenosis was stimulated by the masterly paper of Parker and Weiss in 1936, and has been increased in recent years by the development of surgical treatment of the stenosed valve. In 1949 Larrabee, Parker, and Edwards reasserted the findings of F. Parker and Weiss, and suggested that changes in the vessels might diminish the improvement that could be expected to result from valvotomy. In the series of Brock at Guy's Hospital lingular biopsy was done as a routine in an attempt to correlate the pathological findings with the clinical state and catheter studies of individual patients, and with the general object of increasing our knowledge of mitral stenosis. This was abandoned after 40 consecutive cases when it appeared that the procedure was not entirely harmless to the patient, the most important determining event being the development of a lingular abscess (Brunsdon, Enticknap, and Milstein, 1953). It was then important to take stock of what had been gained, to attempt to evaluate lingular biopsy as a research measure, and to balance the knowledge acquired in individual cases against any possible increased risk of post-operative morbidity.

\section{MATERIAL AND METHODS}

The lingular biopsies were obtained from patients who had undergone cardiac catheterization and mitral valvotomy at Guy's Hospital. Their postoperative course was compared with that of a similar control group of 40 patients whose lungs were left intact. The two groups are not quite comparable as they were not contemporaneous and minor changes in operative technique and management were instituted from time to time. The control series, however, comprises seven patients preceding and 33 patients following the biopsy series, so the effect of these differences is minimized. Four of the patients from whom biopsies were taken died, two within a few hours of operation, one six days, and one eight weeks later.

Method of LUNG Biopsy.-The distal third of the inferior lingular segment was resected imme- diately the chest was opened. The inflated lung was divided between clamps and the cut edge was sutured with two layers of continuous thread. The crushing action of the clamp sealed the edge of the 0 specimen and it was fixed in $10 \%$ formol saline in this condition. Paraffin embedded sections were $\bar{z}$ stained with haemalum and eosin for the general examination and for vascular measurements, and $\stackrel{5}{\square}$ by Perls's method for assessment of haemosiderosis. $\vec{\theta}$ The biopsy and pathological techniques were uni- of form throughout the series so the resulting sections were all of approximately equal size.

The Value of the INVESTigation In Relation to THE INDIVIDUAL PATIENT

This has been assessed first by a comparison of $\stackrel{\mathbb{D}}{\circ}$ the histological findings with the physiological $\overrightarrow{\overrightarrow{0}}$ measurements made at cardiac catheterization, and 3 second by a similar statistical comparison of the degree of haemosiderosis with the amount of radiological soft mottling.

Histo-PHYSIOLOGICAL COMPARISON.-The vascular changes in the sections were compared with pressure measurements by methods previously described (Enticknap, 1953), but only one significant correlation coefficient ( $r$ ) was discovered; this related the mean arteriolar lumen-wall thickness ratio to the mean pulmonary artery pressure at rest and was -0.38 with a standard error (s.e.) of \pm 0.18 . The $\frac{D}{O}$ degree of haemosiderosis was also compared with mean pulmonary artery pressure in 36 cases and it $N$ was found that there was no correlation $(\mathrm{r}=+\mathbf{0 . 0 9}$, s.e. $= \pm 0.18)$.

ASSESSMENT OF HAEMOSIDEROSIS. - Although haemosiderosis of the lungs has long been recognized as a common feature of fatal cases of mitral stenosis, it was only diagnosed when present in $\cong$ extreme degree during life, and was considered of sufficient rarity and interest to be reported as such in the literature (Lancet, 1952).

Increasing acquaintance with the radiographic $\frac{O}{\mathbb{D}}$

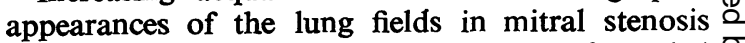
suggested that the very common finding of mottled shadows, apart from the usual vascular streaking, 
might indicate that haemosiderosis was almost universal in patients showing clinical effects of the disease. The diagnosis of haemosiderosis in mitral stenosis is, however, largely a matter of individual standards in radiological interpretation. Lingular biopsy, therefore, offered an opportunity of finding by another method how frequent this change was and correlating the pathological findings with radiological assessment. Clearly if this correlation was exact any disturbance to the patient by lingular biopsy would not be justified on this ground.

The radiological assessment was made by one of us (C.G.B.) and graded from 3 to 0 . As this is an entirely subjective assessment an attempt was made to correlate this with the impressions of others on the following basis:

Grade 3.-Mottling so marked that the diagnosis of miliary tuberculosis, or its differential diagnosis, would be considered on the appearance of the lung fields alone, without the radiological appearance of the heart or clinical findings indicating mitral stenosis. This is the type where haemosiderosis in mitral stenosis was first recognized by clinicians.

Grade 2.-Mottling not so marked yet quite definite; comment would be made by any experienced clinician or radiologist, particularly when the radiological and clinical findings of mitral stenosis drew attention to the lung fields. A definite diagnosis of haemosiderosis would not generally be made.

Grade 1.-Doubtful mottling not easily distinguished from normal vascular markings; comment might be made if the diagnosis of mitral stenosis was in mind, but the lung fields would otherwise be passed as normal.

Grade 0.- No mottling; lung fields would be noted as remarkably clear even for a case of severe mitral stenosis.

In this assessment only widespread mottling was included and it was not confused with the hilar changes of pulmonary venous congestion, nor the increased density and size of the pulmonary artery and branches. The harder larger discrete shadows of similar density to the Ghon focus were noted as a frequent addition to the generalized mottling, but did not increase the grading.

The degree of haemosiderosis was assessed histologically (J.B.E.) also as one of four grades 0 , 1,2 , or 3 , in each case, by evaluating separately the localized form (Lendrum, 1949), and the density of any diffusely distributed siderophores. The severity of each form was marked from 0 to 3 as shown in Table I and the marks were added to give the final grading; one case scored more than three marks but was nevertheless placed in grade 3 .
In these small sections no evidence of calcification nor any other changes that could be equated with the large "hard" shadows described above were seen. Accordingly the degree of haemosiderosis was compared with the radiological assessment of soft mottling in the 29 cases for which suitable radiographs were available and a significant relationship was discovered $(r=+0.42$, s.e. $= \pm 0.19)$. In this series there was, therefore, a reasonable correlation between an individual assessment of haemosiderosis from radiographic appearances and the histological findings of haemosiderosis at lingular biopsy.

T ABLE I

DEGREE OF HAEMOSIDEROSIS

\begin{tabular}{|c|c|}
\hline No. of Marks & Density of Siderophores \\
\hline $\begin{array}{l}0 \\
1 \\
2 \\
3\end{array}$ & $\begin{array}{c}\text { None } \\
\text { A few } \\
\text { Many } \\
\text { Some in every alveolus }\end{array}$ \\
\hline No. of Marks & $\begin{array}{l}\text { Total No. of } \\
\text { Foci in Section }\end{array}$ \\
\hline $\begin{array}{l}0 \\
1 \\
2 \\
3\end{array}$ & $\begin{array}{c}0 \\
1 \text { or } 2 \\
3 \text {, } 4 \\
5 \text { or more }\end{array}$ \\
\hline
\end{tabular}

\section{The Value of Biopsy as a Research Measure}

These specimens have provided unequivocal evidence that marked pulmonary changes occur in cases of mitral stenosis of all grades of disability and are not confined to those which have been in recognized clinical heart failure. Further the excellent fixation of biopsy material has made it possible to study them free from artefact. A sufficient series has, however, accumulated already for the study of the common changes, but to investigate fully the rarel abnormalities many more cases would need to be examined. Thus, in only one of these biopsies was arteriolitis seen, and, as measurements of pulmonary blood pressure are rarely made shortly before death, we may safely draw conclusions about the pathogenesis of this lesion only from biopsy material. Similarly, a search for cases with hyalinization of pulmonary arterioles, most likely to be rewarding in this group in which high pressures are common, would be of little profit if they had not been measured at the time the specimen was obtained. As a third example the observation made on these specimens, that inter-alveolar capillary dilation and arteriolo-sclerosis tend not to occur together, can only be pursued in specimens from patients who are not in circulatory failure. Some observations can, therefore, only be made on biopsy specimens, but if these are to justify 
the procedure it must be shown that the changes in the lingula represent those in the lung as a whole.

It is certain that haemosiderosis is poorly developed in the lingula (Gough,1952), but the correlation found between the histological and radiological findings suggests that the amount in the lingula does bear a constant relation to that elsewhere. The work of Harrison (1953) also suggests that the vascular changes, particularly sclerosis of the large arteries, are less severe in the upper lobes than in the lower. Observations on 35 patients with mitral stenosis examined at necropsy here in the last four years show, however, that while the arteriolar changes sometimes show this distribution, they do not always do so. A semi-quantitative assessment of the degree of vascular abnormality in the 93 available lung lobes from these patients revealed the most marked changes in the lingula and right middle lobe, the six regions falling into the following order: Lingula, R.M.L., L.L.L., R.U.L., R.L.L., L.U.L. The only significant information revealed by statistical treatment of the results was that the lingular and right middle lobes contained a greater amount of arteriolar disease than was likely to have arisen by chance ( $p$ approximately equals 0.04 ) than did the upper lobes. It appears, therefore, that on the whole the lingula is a suitable site for biopsy.

\section{The Possible Harmful Effects of Lingular BiopsY}

To assess these the incidence of the following features was compared in the biopsy and control groups: post-operative pyrexia, number of chest aspirations required, radiographic evidence of lingular opacity, pleural effusions.

The results are shown in Tables II and III.

TABLE II

COMPARISON OF FEATURES OF POST-OPERATIVE COURSE IN "LINGULAR BIOPSY" AND “NO BIOPSY" SERIES

\begin{tabular}{|c|c|c|}
\hline & $\begin{array}{c}\text { Lingular } \\
\text { Biopsy Series }\end{array}$ & $\begin{array}{c}\text { No Biopsy } \\
\text { Series }\end{array}$ \\
\hline $\begin{array}{l}\text { Mean duration of fever (all cases).. } \\
\text { Proportion of cases with fever last- } \\
\text { ing longer than } 2 \text { days } \\
\text { Mean maximum height of fever } \ldots \\
\text { Total no. of chest aspirations } \\
\text { Radiographic lingular opacity }\end{array}$ & $\begin{array}{l}5 \cdot 8 \text { days } \\
53 \cdot 5 \% \\
100 \cdot 3^{\circ} \mathrm{F} \text {. } \\
6 \text { cases }\end{array}$ & $\begin{array}{l}3.7 \text { days } \\
23 \% \\
99.7^{\circ} \mathrm{F} \text {. } \\
1 \\
1 \text { case }\end{array}$ \\
\hline
\end{tabular}

TABLE III

SEVERITY OF PLEURAL EFFUSIONS IN “ LINGULAR BIOPSY" AND "NO BIOPSY" SERIES

\begin{tabular}{|c|c|c|c|c|}
\hline Severity of Effusion & 0 & 1 & 2 & 3 \\
\hline $\begin{array}{l}\text { “ Lingular biopsy" series } \\
\text { “ No biopsy " ser ies }\end{array}$ & $\begin{array}{r}7 \\
13\end{array}$ & $\begin{array}{r}6 \\
10\end{array}$ & $\begin{array}{r}13 \\
3\end{array}$ & 2 \\
\hline
\end{tabular}

Post-operative Pyrexia.-The period of post- $\overrightarrow{\vec{A}}$ operative fever was measured from the first post- $\overline{0}$ operative day to the first day that the temperature $\frac{c}{0}$ became normal and remained so. In cases where $\overline{\bar{c}}$ the temperature, having become normal, rose again, $\overrightarrow{\mathbb{D}}$ the first period of pyrexia was used in the calculations. The mean duration of fever was rather longer $c$ in the biopsy group but this difference was not $\vec{\circ}$ significant. However, as fever lasting 48 hours is $\overrightarrow{-}$ common after many operations it might be expected $\omega$ that the inclusion of such cases in both groups would obscure a significant difference. That this is the case is suggested by the fact that the biopsy group does contain significantly more cases with a ir fever of more than three days' duration than does the control group $(\mathrm{p} \bumpeq 0.04)$.

The mean maximum post-operative fever was also higher in the lingular biopsy group, and, although @ the difference in the two groups did not reach $\stackrel{3}{\rightarrow}$ significant limits, the tendency was again to the $\vec{\theta}$ advantage of the control group.

RADIOGRAPHIC EVIDENCE OF LINGULAR OPACITY. -In some of the cases in which lingular biopsy was performed, an opacity was noted in the region of the inferior lingular segment in radiographs taken about a week after operation. This shadow, irre-

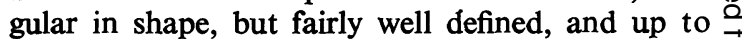
$2 \mathrm{~cm}$. in diameter, probably represents an area of $\overrightarrow{0}$ haemorrhagic pneumonitis in the lung adjacent to 3 the suture line. It was seen in eight cases in the lingular biopsy group, but was almost certainly present in many more, in which pericardial and pleural effusions obscured the appearance of the lung parenchyma. The only lingular opacity seen in the control group was probably due to an 3 infarct. The lingular shadow usually persisted for a week or two, but in one case it persisted for over a month (Fig. 1).

Pleural Effusion.-The pleural effusions as seen on the available post-operative radiographs were graded arbitrarily according to their extent and duration into four groups, ranging from 0 to $3 . \Omega$ Table III shows that the effusions were more severe $N$ in the lingular biopsy group and that severe effusions $N$ of grades 2 and 3 were significantly more common $(0.02>\mathrm{p}>0.01)$. The table shows the total number of aspirations required in each group, and not $\frac{}{\Phi}$ aspirations per case. This procedure was not often $\stackrel{9}{?}$ necessary, but was more often required in the lingular biopsy group.

Morbid Anatomical Changes at the Biopsy Site $\frac{\stackrel{\rho}{\mathbb{D}}}{\varrho}$

Five cases necropsied after intervals of fromo 12 hours to nine weeks after valvotomy and biopsy, 


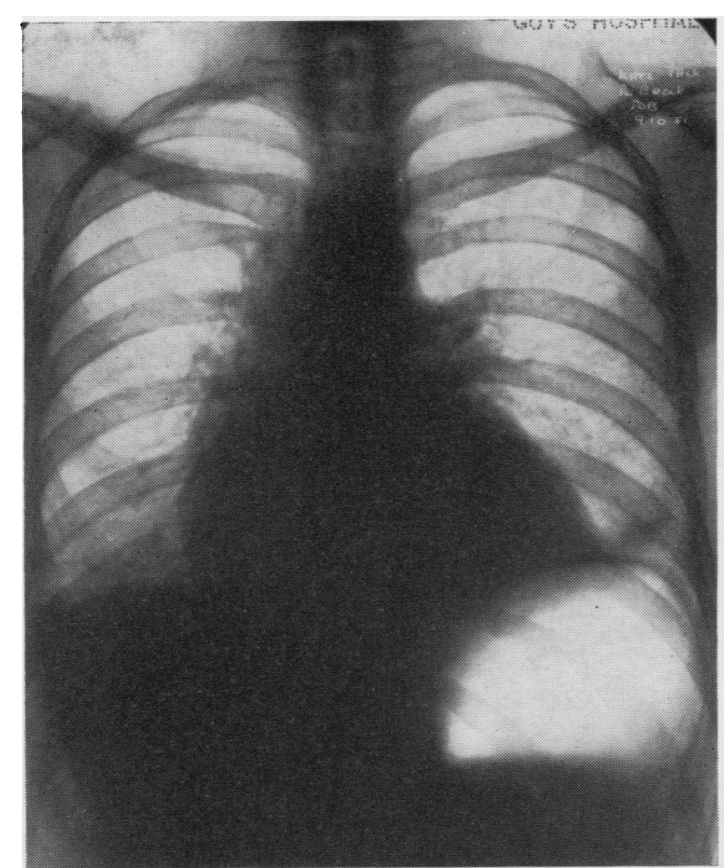

FIG. 1.-Reduced radiograph of lung fields of male patient aged 37 , one month after valvotomy, showing opacity at site of lingular biorsy.

four of them from this series, provide some direct evidence on the nature of these changes. In two of them, dying 12 hours and 60 hours after operation, inspection of the biopsy site with the naked eye revealed no abnormality, but histological examination was not made. In one patient who lived six days, however, histological sections show an area of lung necrosis involving the distal $1 \mathrm{~cm}$. of the remaining part of the lingula (Fig. 2); there is no evidence of infection, only a little haemorrhage, and the vessels leading to the necrotic area are thrombosed. A further case, not of this series, died from a broncho-pleural fistula six weeks after operation, and presented a less clear-cut picture. It is certain that the fistula, which was in the anterior segment of the left upper lobe, was not at the site of the biopsy, but it was associated with such a widespread pneumonia of the whole lobe that the healing of the lung incision could not be assessed. In the last relevant case, a patient who survived eight weeks, the biopsy specimen had shown a considerable degree of both acute and chronic sepsis; at necropsy an actual abscess cavity was found to have formed in relation to the thread suture (Brunsdon and others, 1953).

\section{Discussion}

From all these observations certain conclusions may be drawn. Radiological demonstration of soft mottling agreed fairly well with the actual degree of haemosiderosis in the biopsy. As a research measure the biopsies promise to answer some questions that will be difficult or impossible to answer by any other method. Although there is some evidence that the lingula is not a fair sample of the lung, it is on the whole quite satisfactory for the purpose. There were definite disadvantages to the patient when lingular biopsy was performed by the technique described during mitral valvotomy. Post-operative effusions were more prominent, more often requiring aspiration, and were associated with a more prolonged pyrexia. No new evidence of clinical value was gained. The information derived from the biopsy was of no assistance in assessing immediate prognosis, and the state of the vessels could in some measure be deduced from the relationship shown to exist between them and the pulmonary blood pressure as measured by cardiac catheterization. This can, in any case, be roughly estimated by clinical methods.

The cause of the increased effusions may be found in the technique that was used. It could be argued that if clamps had not been employed and the lung had been sutured by non-reactive suture material such as nylon, this complication

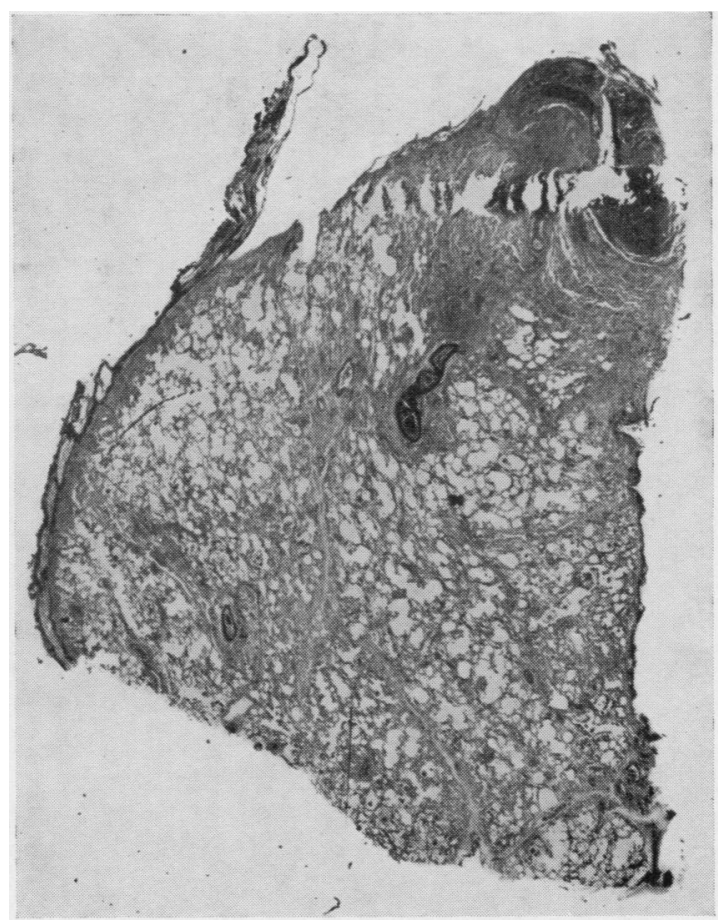

Fig. 2.-Antero-posterior section of lingular biopsy site six days after operation, showing necrosis in relation to suture material, the position of which is shown by the break in the section. (Haematoxylin and eosin $\times 4$.) 
would not have occurred. On the other hand some degree of pulmonary infection may be present in the cases submitted to operation and the effusions may be due to contamination of the pleural cavity following the incision. This contamination would probably have been greater if clamps had not been used. Furthermore, the same technique has been used for lung biopsy in a variety of different pulmonary conditions and it is our impression that it has not there caused pleural effusions. It seems, therefore, more probable that the development of these effusions is dependent on the changes produced in the lung by mitral stenosis. The most important of these is chronic congestion, evidence of which is frequently seen at operation, and acute congestion following operation could have similar effects.

The nature of the lesions causing the lingular opacities is doubtful, but they probably contribute to the degree of effusion. The number of cases examined at necropsy is too few to justify a detailed account of the healing of the lingula following resection for biopsy in this disease, and those recorded here probably present an unduly pessimistic view of this process. Nevertheless, they represent all the data at present available and do make it clear that straightforward healing is not invariable. As the radiographic and clinical data indicate the frequent development of a moderate sized lesion, the fact that necrosis of the clamped area has actually been demonstrated makes it probable that it is the usual course. If this is so, infection of the necrosed area is occasionally to be expected, and this will occur particularly in those cases with antecedent lung infection which may, as in our last case, be reflected in the histology of the biopsy specimen.

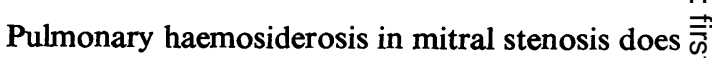
not appear to correlate with haemoptysis, the degree of pulmonary hypertension, or give any indication $\frac{C}{0}$ of the severity or prognosis of the disease. Little $\overline{\frac{\rho}{3}}$ is known of this frequent finding and light might $ه$ well be shed on it if biopsy were continued. There does not appear to be any correlation between the ${ }^{\infty}$ degree of lingular sepsis and the incidence of $\vec{O}$ complications, nor did numerous calculations of $\overrightarrow{ }$ correlations between clinical and histological $\omega_{\mathscr{N}}$ methods of assessing prognosis reveal any signi- $\frac{\vec{z}}{\times}$ ficant data.

\section{SUMMARY}

The progress following valvotomy in 40 consecu- id tive patients with mitral stenosis in whom lingular 을 biopsy was done has been compared with a similar control group.

Lingular biopsy was associated with more marked pleural effusions and more pyrexia.

Necrosis of the biopsy site has been observed $\overrightarrow{0}$ and in one patient a localized abscess developed.

A correlation was found between the histological and radiological assessments of haemosiderosis.

Because of the morbidity resulting from lingular biopsy it should not lightly be performed as a routine, but it can be justified as a research measure $\stackrel{\circ}{\varnothing}$ that may eventually benefit the large numbers of $\triangle$ patients who suffer from mitral stenosis.

REFERENCES

Brunsdon, D. F., Enticknap, J. B., and Milstein, B. B. (1953). Guy's Hosp. Rep., 102, 303.

Enticknap, J. B. (1953). J, clin, Path., 6, 84

Gough, J. (1952). Personal communication.

Harrison, C. V. (1953). Communication to the Pathological Society arrison, C. V. (1953). Communich
of Great Britain and Ireland.

Lancet (1952). 1, 1005.

Larrabee, W. F., Parker, R. L., and Edwards, J. E. (1949). Proc. Mayo Clin., 24, 316.

Lendrum, A. C.'(1950). J. Path. Bact., 62, 555.

Parker, F., and Weiss, S. (1936). Amer. J. Path., 12, 573. 